\title{
Hemidiaphragmatic paralysis and hemohydrothorax following right internal jugular vein catheterization
}

\author{
In-Suk Kwak, Gang-Seuk Riew, Ji Young Bae, Tae-Wan Lim, and Kwang-Min Kim \\ Department of Anesthesiology and Pain Medicine, Hangang Sacred Heart Hospital, College of Medicine, Hallym University, Seoul, Korea
}

21 year-old female patient $(167 \mathrm{~cm}, 80 \mathrm{~kg}$ ) has decided to undergo radical free flap in the left leg. After anesthetic induction, the needle was inserted by an anterior approach for central venous catheterization in right internal jugular vein. Good aspiration of blood into the syringe was seen at second venipuncture. One milliliter of blood was aspirated out and intravenous (IV) fluids were injected through a 14 gauge (G) main catheter after insertion of the catheter.

After confirming the position of the patient over two steps (from supine to prone and right lateral decubitus) aspiration of blood through side catheter (18 G) was not done well and only injection of IV fluids was possible. We connected $500 \mathrm{ml}$ $6 \%$ hydroxyethyl starch (Voluven, Fresenius Kabi, Germany) to each central venous catheter (CVC), because ineffective back flow was thought to be caused by obesity and right lateral decubitus position of the patient. The central venous pressure was measured ( $8 \mathrm{mmHg}$ ) and a wave pattern was seen. Three hours in the surgery, the patient again exhibited spontaneous respiration so $10 \mathrm{mg}$ of rocuronium was injected through $18 \mathrm{G}$ catheter of the central venous catheter. However, spontaneous respiration continued so another $10 \mathrm{mg}$ of rocuronium was injected. Still, spontaneous respiration did not disappear and another $20 \mathrm{mg}$ of rocuronium was injected but it had no effects. So we suspected the CVC was not placed in the vessel. Aspiration via CVC was immediately done and 2,400 $\mathrm{ml}$ of IV fluids were drained. The position of the CVC was to be checked after the operation and the surgery was continued after securing IV fluid supply line by the peripheral vessels.
The postoperative CT of the neck and chest showed collapse of the right internal jugular vein, and the CVC running lateral to the vein, and the tip of the catheter was found in the pleural cavity (Fig. 1).

The endotracheal tube was removed on postoperative day 2 and the chest tube was removed after 9 days. Elevation of right diaphragm was found in the chest x-ray after 2 weeks but the patient was asymptomatic. The patient was under observation for 1 week but the paralysis did not improve. Fluoroscopy of diaphragm was done and it showed no movement of right diaphragm so the right diaphragmatic paralysis was diagnosed. The progress of the paralysis was observed because the patient did not have any complaining symptoms. The patient was discharged on post-op day 32. The elevated right diaphragm still remained in the chest $x$-ray before the discharge but it improved after 6 months.

Because it is uncommon to find hydrohemothorax with diaphgragmatic paralysis among the complications associated with a CVC, a similar case could not be found. In addition, there are two points in this case that must be carefully looked at. First, that insertion of catheter outside the vessel could not be detected during insertion process even though there was about $1 \mathrm{ml}$ of blood regurgitation. Second, the causes of the diaphragmatic paralysis was not clear.

Hydrohemothorax or hydromediastinum can occur when IV fluids are injected without detecting malposition of the CVC. The following examples have been reported: when the catheter is placed outside the vessel as the vessel is expanded by a guide-

Corresponding author: Ji Young Bae, M.D., Department of Anesthesiology and Pain Medicine, Hangang Sacred Heart Hospital, College of Medicine, Hallym University, Yeongdeungpo-dong 2-ga, Yeongdeungpo-gu, Seoul 150-719, Korea. Tel: 82-2-2639-5507, Fax: 82-2-2631-4387, E-mail: baejjy@naver.com

(c) This is an open-access article distributed under the terms of the Creative Commons Attribution Non-Commercial License (http:// creativecommons.org/licenses/by-nc/3.0/), which permits unrestricted non-commercial use, distribution, and reproduction in any medium, provided the original work is properly cited. 

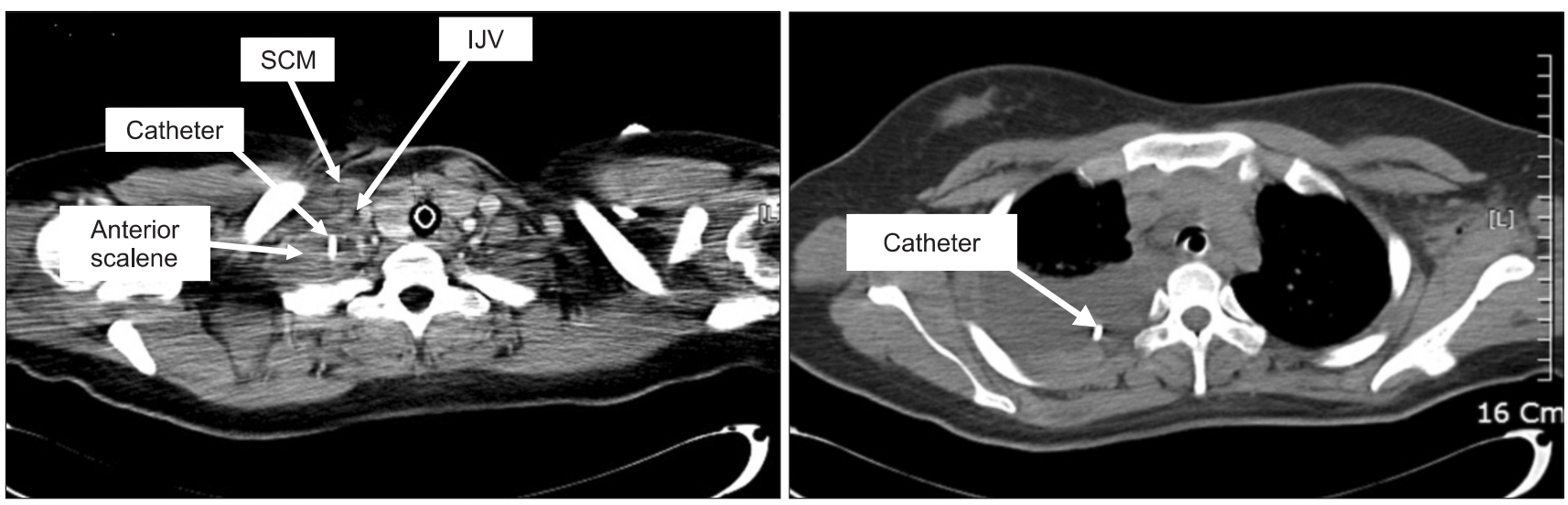

Fig. 1. Chest and neck CT with enhancement. Right internal jugular vein is collapsed and the edematous anterior scalene muscle contains the catheter. The catheter is in the pleural cavity. IJV: internal jugular vein, SCM: sternocleidomastoid muscle.

wire or dilator during the process of inserting the CVC [1]; when the vein ruptures due to excess IV fluids after the catheter was inserted in the external jugular vein or bracheocephalic vein rather than the targetted central vein [2]; and when the catheter deviates outside the vessel due to change of vessel wall or because the catheter is not fixed well in place even after placement of the catheter within the targetted vein [3].

The chest CT of the patient showed the CVC passed down outside the internal jugular vein into the pleural cavity. So primary malposition with initial intrapleural placement seems to be more likely. During catheterization, we confirmed aspiration of blood into the transparent part of large bore lumen and easy flushing indicated correct intrvascluar catheter placment. However, Hohlrieder et al. [4] mentioned that the blood regurgitated up to the transparent part of the $12 \mathrm{Fr}$ catheter even though the CVC malposition, and described that $0.1 \mathrm{ml}$ of blood can fill about $1 \mathrm{~cm}$ of the transparent part of $12 \mathrm{G}$ catheter. The CVC used in the case that the authors read had volume less than $1 \mathrm{ml}$ within the catheter. In this patient, hemorrhage occurred because the wall of vein was perforated by the guidewire or dilator. If the catheter was filled with the blood due to more than $2 \mathrm{ml}$ hemorrhage and the tip of the catheter was sunk where the blood was collected, about $1 \mathrm{ml}$ of blood could have been aspirated.

The possible causes of the right diaphragmatic paralysis are phrenic nerve injury due to needle puncture in the neck, and phrenic nerve compression due to edema in the muscles and soft tissues around the catheter placed outside the vein. Also, inflammation due to injection of excess IV fluids may have caused the delayed diaphragmatic paralysis. No clinical reports of inflammation of colloids or crystalloids within the pleural cavity were found, but Hydroxyethyl starch solution mixed with blood was described to activate neutrophils and increase their adhesion ex vivo [5].

In order to reduce these complications, presence of catheter outside the vein needs to be detected immediately by aspirating enough blood through all catheters as well as the main catheter even with a successful venipuncture. And the additional control aspiration needs to be checked frequently during surgery before starting the infusions.

\section{References}

1. Kim YO, Lee JR, Kim KT, Choi WJ, Lee SI, Kim JW. Hydrothorax after central vein catheterization for right internal jugular vein and contralateral reexpansion pulmonary edema after right chest tube insertion - A case report. Korean J Anesthesiol 2007; 53: 234-7.

2. Kwak HJ, Lim ES, Ban SY, Lee JY, Yoon JS, Kil HK, et al. Hydromediastinum following Internal Jugular Vein Catheterization - A case report. Korean J Anesthesiol 2007; 52: 335-8.

3. Kunizawa A, Fujioka M, Mink S, Keller E. Central venous catheterinduced delayed hydrothorax via progressive erosion of central venous wall. Minerva Anestesiol 2010; 76: 868-71.

4. Hohlrieder M, Schubert HM, Biebl M, Kolbitsch C, Moser PL, Lorenz IH. Successful aspiration of blood does not exclude malposition of a large-bore central venous catheter. Can J Anaesth 2004; 51: 89-90.

5. Rhee P, Wang D, Ruff P, Austin B, DeBraux S, Wolcott K, et al. Human neutrophil activation and increased adhesion by various resuscitation fluids. Crit Care Med 2000; 28: 74-8. 